22 Young RJ, Macintyre CCA, Martyn CN, et al. Progression of sub-clinical polvneuropathy in voung patients with type 1 diabetes. Associations with glycaemic control and microangiography. Diabetologia 1986;29:156-61

23 Dyball REJ, Tate PA. Basic embryology and the embryological basis of malformation syndromes. In: Robertson NEC, ed. Textbook of neonatology. malformation syndromes. In: Robertson NEC

24 Sadler TW, Horton WE, Hunter ES. Mechanisms of diabetes-induced congenital malformations as studied in mammalian embryo culture. In: Jovanovic L, Peterson CM, Fuhrmann K, eds. Diabetes and pregnancy, teratology, toxicity and treatment. New York: Praeger, 1987:51-71.

25 Rayburn W, Piehl E, Jacober S. Severe hypoglycaemia during pregnancy, its frequency and predisposing factors in diabetic women. Int $\mathcal{G}$ Gynaecol Obstet 1986;24:263-8.

26 Bergman M, Seaton TB, Averhahn CC, et al. The incidence of gestational hypoglycemia in insulin-dependent and non-insulin dependent diabetic women. N Y State f Med 1986;86:174-7.
27 Bergman M, Newman S. Hypoglycemia in pregnancy, unknown risks. Diabetes Care 1987:10:180.

28 Mills JL, Knopp RH, Simpson JL, et al. Lack of relation of increased malformation rates in infants of diabetic mothers to glycemic control during organogenesis. N Engl f Med 1988;318:671-6.

29 Anonymous. Congenital abnormalities in infants of diabetic mothers [Editorial]. Lancet 1988;i:1313-5.

30 Fuhrmann K, Reiher H, Semmler K, et al. Prevention of congenital malformation in infants of insulin dependent diabetic mothers. Diabetes Care 1983;6:219-23.

31 Fuhrmann K, Reiher H, Semmler K, Glockner E. The effect of intensified conventional insulin therapy before and during pregnancy on the malformation rate in offspring of diabetic mothers. Exp Clin Endocrinol 1983;83: 173-7.

(Accepted 8 August 1990)

\title{
Ultrasonography compared with intravenous urography in the investigation of adults with haematuria
}

\author{
John Spencer, David Lindsell, Irene Mastorakou
}

\begin{abstract}
Objective-To compare ultrasonography with intravenous urography in the investigation of adults with haematuria.

Design-Prospective study entailing the examination of all patients with both investigations concurrently. The investigations were performed independently on routine lists by different duty radiologists. Each was aware of the details of the request form but not of the findings of the other investigation.
\end{abstract}

Setting-Radiology department of a teaching hospital.

Patients-155 Consecutive adult patients (aged 18-93) referred from general practitioners and hospital outpatient clinics with a history of haematuria.

Follow $u p-$ When results of both examinations proved normal no clinical or radiological follow up was sought. All abnormal findings of either investigation were correlated with results of subsequent imaging studies or operative findings.

Results -81 Patients (52\%) had normal findings on urography and ultrasonography. Overall, the findings of ultrasonography concurred with those of urography in 144 cases (93\%). Among the discrepant findings of the two investigations ultrasonography missed two ureteric calculi; one was in a non-dilated ureter, and in the other case ultrasonography detected the secondary ureteric dilatation. Ultrasound examination alone detected four bladder tumours not visible on urography with sizes ranging from 5 to $21 \mathrm{~mm}$, representing one fifth of the 20 cystoscopically proved bladder tumours detected in the series. Ultrasonography detected all the 22 neoplastic lesions discovered in the study (20 bladder, two renal). Ultrasonography clarified the nature of renal masses evident in three urograms (simple cysts).

Conclusions-Ultrasonography is a safe and accurate method of investigating the urinary tract in adults with haematuria. When combined with a single plain abdominal radiograph it proved to be superior to urography as the primary imaging study in this series. Ultrasonography should certainly be preferred to urography if cystoscopy is not planned. No urothelial tumours of the upper urinary tract were found in the series, reflecting their rarity. For those patients in whom ultrasonography and plain radiography have shown no abnormality and in whom cystoscopic appearances are normal urography would be advisable to exclude urothelial tumours of the upper urinary tract.

\section{Introduction}

It has been standard urological practice to request an intravenous urogram as the initial radiological investigation of patients with haematuria. In the light of improvements in newer imaging techniques the continued use of urography has been questioned. ${ }^{1}$ Ultrasonography can provide images of both the upper and lower urinary tract and is without the potential hazards of ionising radiation and intravenous contrast media. This technique supplemented by a single abdominal radiograph has supplanted urography in investigating certain urinary tract disorders, notably urinary tract infection in childhood, ${ }^{23}$ and a similar policy has been recommended for the investigation of adults with urinary tract infection. ${ }^{+}$Ultrasonography has also been shown to have advantages over urography in assessing prostatic disease. ${ }^{56}$ The efficacy of ultrasonography in the investigation of haematuria does not seem to have been assessed prospectively in a large group of adults. We therefore conducted a comparative study of the accuracy of ultrasonography and urography in patients with haematuria referred for urography from both general practice and hospital outpatient clinics.

\section{Patients and methods}

One hundred and fifty five consecutive patients (121 men, 34 women) referred from general practitioners and the hospital outpatient department with a history of haematuria and a request for intravenous urography were entered into the study. Their ages ranged from 18 to 93 years (mean 59.7), and most were referred from general practice. The clinical details provided on the request forms for some patients were very limited and did not indicate the type or severity of haematuria in all cases. We did not include patients with haematuria associated with a known urinary tract infection. These patients were entered into a separate prospective comparative trial. ${ }^{4}$ Patients with traumatic haematuria and those with acute ureteric colic were also excluded.

The patients were sent a routine appointment for intravenous urography with an explanation that an ultrasound examination would also be performed. In all cases the investigations were performed concurrently. The patients attended the hospital between October 1988 and January 1990. 
A mild laxative was given the night before urography and the patient starved from midnight. A full length abdominal radiograph was taken initially with supplementary views when appropriate. An intravenous injection was then given of either meglumine diatrizoate and sodium diatrizoate ( $325 \mathrm{~g}$ iodine/l) or iopamidol $(300 \mathrm{~g} / \mathrm{l})$ when indicated at a dose of $1 \mathrm{ml} / \mathrm{kg}$ body weight. Immediately after the injection an upper abdominal (cross kidney) radiograph was taken followed by a similar film five minutes after injection. (At the discretion of the supervising radiologist renal area tomograms were taken or abdominal compression applied after inspection of these initial radiographs.) A full length abdominal radiograph was taken roughly 20 minutes after injection, with a view of the bladder when full. The ultrasound examination was then performed and the patient returned to the urography room for postmicturition radiography of the bladder.

The ultrasound examination was performed with either an Accuson 128 or an ATL Ultramark 4 machine using a $3.5 \mathrm{MHz}$ sector transducer. A routine transabdominal examination of the urinary tract was made in the supine position before returning the patient for completion of the urogram. The bladder was not filled specifically for the ultrasound examination.

The two investigations were reported independently. The reports of the two examinations that were issued to the referring clinician were then compared and the results correlated by us. When no further investigation was planned after the clinical assessment, urography, and ultrasound examination had failed to detect anything abnormal a diagnosis of "normal" was assumed. These patients will be the subject of a long term follow up study. When the two investigations were in agreement about the nature of abnormalities and no further investigations were indicated or planned the diagnosis was accepted. When a discrepancy was discovered the investigations were reviewed. The eventual diagnosis was made with reference to additional clinicopathological data or imaging studies, or both, or from the findings at cystoscopy or other operative procedures when performed. All cases of neoplasia diagnosed by either examination were correlated with findings at cystoscopy or laparotomy and subsequent pathology reports.

\section{Results}

Eighty one $(52 \%)$ of the 155 patients examined had normal urograms and ultrasonograms. Table I lists the abnormalities detected by urography. Eighty abnormalities were found in 74 patients at urography, but in 11 cases urography was unable to determine the exact cause (six renal space occupying lesions, three minor unilateral hydronephroses, a parapelvic mass, an

TABLE I - Findings of intravenous urography in 155 patients

\begin{tabular}{lc}
\hline & No \\
\hline Normal findings & 81 \\
Prostatic enlargement & 30 \\
Bladder neoplasm & 16 \\
Urinary calculi with or without hydronephrosis & 12 \\
Renal & 8 \\
Ureteric & 3 \\
Bladder & 1 \\
Renal space occupying lesion & 6 \\
Unexplained unilateral hydronephrosis & 3 \\
Bladder diverticulum & 3 \\
Bilateral hydronephrosis (outflow obstruction) & 2 \\
Renal neoplasm & 2 \\
Medullary sponge kidney & 2 \\
Atrophic kidneys & 2 \\
Parapelvic mass & 1 \\
Filling defect in right upper pole calix & 1 \\
\hline Total abnormalities discovered & 80
\end{tabular}

upper pole caliceal filling defect). The most common abnormal finding, prostatic enlargement, was suggested in most cases as the likely diagnosis by the referring doctor on the basis of clinical features but was better delineated by ultrasonography. Ultrasonography detected 75 of the 80 abnormalities (94\%) identified by urography. Table II gives the findings in cases in which ultrasonography and urography gave different results. In addition to clarifying the nature of the seven renal

TABLE II -Cases in which findings at urography and ultrasonography differed (11 patients)

\begin{tabular}{|c|c|}
\hline & No \\
\hline \multicolumn{2}{|l|}{ Shown by intravenous urography alone: } \\
\hline Ureteric calculi (visible on plain film) & $2^{\star}$ \\
\hline Bladder diverticulum (patients with enlarged prostate) & 2 \\
\hline Filling defect in right upper pole & 1 \\
\hline \multicolumn{2}{|l|}{ Shown by ultrasonography alone: } \\
\hline Bladder neoplasms & 5 \\
\hline $\begin{array}{l}\text { True positive } \\
\text { S }\end{array}$ & 4 \\
\hline False positive & 1 \\
\hline Renal stone & $1+$ \\
\hline
\end{tabular}

^In one case ultrasonography showed the secondary upper tract dilatation. †Not seen in abdominal radiographs.

TABLE III-Agreement between reported findings of urography and ultrasonography (155 patients)

\begin{tabular}{lc}
\hline & No \\
\hline $\begin{array}{l}\text { Normal on ultrasonography; normal on intravenous } \\
\text { urography }\end{array}$ & 81 \\
$\begin{array}{l}\text { Same abnormality detected by intravenous urography and } \\
\text { ultrasonography }\end{array}$ & 65 \\
$\begin{array}{c}\text { Additional minor information provided by intravenous } \\
\text { urography` }\end{array}$ & 2 \\
$\begin{array}{c}\text { Normal on ultrasonography; abnormal on intravenous } \\
\text { urography }\end{array}$ & 3 \\
$\begin{array}{c}\text { Abnormal on ultrasonography; normal on intravenous } \\
\text { urography }\end{array}$ & 6 \\
\hline
\end{tabular}

*Small bladder diverticula in patients with enlarged prostates.

and parapelvic lesions, ultrasonography showed four bladder neoplasms missed by urography (size range $5-21 \mathrm{~mm}$ ). In one case ultrasonography was reported as showing a $20 \mathrm{~mm}$ bladder neoplasm which was not seen at cystoscopy and which was thought likely to have represented a thickened bladder fold.

Two of the five abnormalities missed by ultrasonography were small bladder diverticula in patients with prostatic enlargement and thus were incidental. The pelvicaliceal filling defect was assumed to have represented a blood clot as it was not present in a follow up urogram. The underlying abnormality remained obscure after urological assessment. The two ureteric calculi that were missed by ultrasonography were both visible on the plain abdominal radiographs, though in one case ultrasonography detected the secondary hydroureter.

Four $(15 \%)$ of the 27 patients aged 40 or less had abnormal findings, and in one of these with a small bladder papilloma the abnormality was visible only with ultrasonography. Ultrasonography and a single plain abdominal radiograph also detected the other three abnormalities discovered by urography in this group of patients (two urinary calculi, one medullary sponge kidney).

Overall, ultrasonography and urography agreed in their reported findings in 144 of the 155 patients studied $(93 \%)$, though in some cases one or other examination showed an abnormality better. Table III gives an overall comparison of the issued reports of urography compared with those of ultrasonography.

\section{Discussion}

The patients referred for investigation in this study had haematuria varying in severity from dipstick or 
microscopic detection to frank haematuria and reflected the broad range of patients referred for radiological assessment, particularly from general practice. The frequency of microscopic haematuria in screened populations has been reported at up to $13 \%$, of whom only $2 \cdot 3 \%$ were found to have "serious urological disease" and less than $1 \%$ to have bladder or renal cancer. ${ }^{7}$ A combination of ultrasonography and flexible cystoscopy has recently been suggested in assessing elderly men discovered to have haematuria on dipstick testing.

One of the aims in investigating haematuria is to diagnose treatable neoplasms of the urinary tract. Most will be bladder neoplasms, ${ }^{9}$ and in our series ultrasonography was superior to urography in diagnosing these. The value of ultrasonography in diagnosing bladder tumours is established, ${ }^{10}$ and the technique has been suggested as an alternative to cystoscopy in detecting recurrent tumours. ${ }^{11}$

Ultrasonography detected the two renal cell carcinomas in our series and clarified the nature of renal masses found at urography. The role of ultrasound examination in assessing renal masses is well established. ${ }^{2}$ Ultrasonography has been shown to be superior to urography in the detection of renal masses, particularly those of less than $2 \mathrm{~cm}$. $^{13}$

Ultrasonography, however, is at a disadvantage compared with urography in that the normal ureter is not identified and the anatomical detail obtained of the renal pelvis is inferior. Urothelial tumours of the upper urinary tract are rare compared with bladder tumours, ${ }^{1415}$ and none were encountered in our series. Most ureteric tumours present with upper tract dilatation, which would be identified by ultrasonography and indicate the need for further investigation. The urographic findings of renal pelvic tumours in previous series suggest that ultrasonography would be inferior to urography in diagnosing them. Over two thirds were simply represented in urograms by filling defects in the pelvicaliceal system..$^{1516}$

In up to a quarter of cases investigated haematuria occurs as a result of urinary tract infection. ${ }^{9}$ We have previously shown that ultrasonography and a single abdominal radiograph are of similar accuracy to urography for investigating adults with urinary tract infection, ${ }^{4}$ and this conclusion extends to the subgroup whose infection is attended by haematuria (Spencer et al, unpublished).

Calculi in the urinary tract account for up to one fifth of cases of haematuria. ${ }^{9}$ Ultrasonography diagnosed all the cases of renal and bladder calculi in our series. Ultrasonography has previously been shown to be comparable to plain radiography in accuracy for diagnosing renal calculi. ${ }^{17}$ We believe that ultrasonography remains at a disadvantage in diagnosing small stones in the ureter and advocate the addition of a plain abdominal radiograph to improve their detection. Urography remains the preferred investigation in acute renal or ureteric colic because in early or subacute ureteric obstruction there may be no proximal dilatation despite reduced or absent excretion of contrast medium from the kidney at urography. ${ }^{18}$

In our series ultrasonography proved superior to urography, notably because of its ability to detect small bladder tumours not visible in urograms. The deficiency of ultrasonography in diagnosing ureteric calculi was correctable by adding a single abdominal radiograph. It is thus tempting to advocate replacing urography by ultrasonography and a plain film in the investigation of adults with haematuria. In addition to a reduction in the hazards to patients from ionising radiation and injected contrast media consequent on such a change, considerable savings would ensue. Even with conservative costings of the consumables used in the two examinations (injection materials and intra- venous contrast, radiographic and photographic film) the cost of a urogram is roughly 10 times that of an ultrasound examination and plain film. ${ }^{19}$

There are some settings, however, in which urography remains essential. We advocate the continued use of urography in patients with proved cytological findings indicating a urothelial neoplasm as an upper tract tumour would be better seen on urography and cystoscopy would usually be performed in such cases. A urogram should be obtained in patients with haematuria in whom cystoscopy has shown nothing abnormal. Urography should be used for assessing the upper urinary tracts in patients with a history of bladder neoplasia because of the recognised association with transitional cell tumours of the ureter or renal pelvis. ${ }^{1+16}$ Conversely, because of the rarity of these upper urinary tract urothelial tumours compared with bladder tumours all patients examined for haematuria should have at the very least an ultrasound scan of their bladders included in their radiological investigation, especially when cystoscopy is not contemplated. The discovery of a suspicious lesion in the bladder by ultrasonography is an indication for urgent urological assessment and may expedite cystoscopy.

The decision to move from urography to ultrasonography as the primary imaging study in a particular centre will be influenced as much by the needs of referring clinicians as by the overall accuracies of two techniques available to the radiologist. The operator dependence of ultrasonography is well recognised and therefore any change in imaging protocol will depend on local skill. In our study the ultrasound examinations were performed by several different operators with varying experience of the technique. The results of the study do not reflect solely the skill of a single radiologist with a particular interest in ultrasonography.

We thank all of our colleagues who peformed investigations as part of this study. We are grateful to $\mathrm{Mr} \mathrm{G}$ Fellows, $\mathrm{Mr}$ J Smith, and Dr E W Fletcher for reading the manuscript and for helpful advice.

1 Corwin HL, Silverstein MD. The diagnosis of neoplasia in patients with asymptomatic microscopic hematuria: a decision analysis. $\mathcal{F}$ Urol 1988;39. $1002-6$.

2 Alon U, Menucha P, Davidai G, Berant M. Ultrasonography in the radiological evaluation of children with UTI. Pediatrics 1986;78:58-64

3 Lindsell D, Moncrieff $M$. Comparison of US examination and intravenous urography after urinary tract infection. Arch Dis Child 1986;61:81-3.

4 Spencer J, Lindsell D, Mastorakou I. Ultrasound compared with intravenous urography in the investigation of adult urinary tract infection. $B M \mathcal{J}$ 1990;301:221-4.

5 Lucas MG, Bodley RN, Lindsell DRM, Fletcher EWL, Scott NP. Urography prior to prostatectomy. Postgrad Med f 1986;62:1117-9.

6 Fidas A, McKinley J, Wild SR, Chisholm GD. Ultrasonography as an alternative to IVU in prostatism. Clin Radiol 1987;38:479-81.

7 Mohr DN, Offord KP, Owen RA, Melton LJ. Asymptomatic microhematuria and urologic disease: a population based study. FAMA 1984;256:224-9.

8 Britton JP, Dowell AC, Whelan P. Dipstick haematuria and cancer in men over 60: results of a community study. BMF 1989;299:1010-2.

9 Abuelo JG. The diagnosis of haematuria. Arch Intern Med 1983;143:967-70.

10 Abu-Yousef MM, Narayana AS, Franken EA, Brown RC. Urinary bladder tumors studied by cystosonography. Radiology 1984;153:223-6.

11 Davies AH, Cranston D, Meagher T, Fellows GJ. Detection of recurrent bladder tumours by transrectal and abdominal ultrasound compared with cystoscopy. Brf Urol 1989;64:409-11.

12 Jacobson HG, Goldberg BB. The role of ultrasonography in the diagnosis of the renal mass and impaired renal function. FAMA 1984;251:2561-3.

13 Warshauer DM, McCarthy SM, Street L, et al. Detection of renal masses: sensitivities and specificities of excretory urography/linear tomography, US and CT. Radiology 1988;169:363-5.

14 Williams CB, Mitchell JP. Carcinoma of the ureter-a review of 54 cases. Brf Urol 1973;45:377-87.

15 Williams CB, Mitchell JP. Carcinoma of the renal pelvis - a review of 43 cases. Brf Urol 1973;45:370-6.

16 Grace DA, Taylor WN, Taylor JN, Winter CC. Carcinoma of the renal pelvis: a 15 year review. F Urol 1967;98:566-9.

17 Middleton WD, Dodds WJ, Lawson TL, Foley WD. Renal calculi: sensitivity for detection with US. Radiology 1988;167:239-44.

18 Erwin BC, Carroll BA, Sommer FG. Renal colic: the role of ultrasound in initial evaluation. Radiology 1984;152:147-50.

19 Lewis-Jones HG, Lamb GHR, Hughes PL. Can ultrasound replace the intravenous urogram in the preliminary investigation of renal tract disease. Brf Radiol 1989;62:977-80.

(Accepted 7 September 1990) 